\title{
Deep Into the Fibers! Postmortem Diffusion Tensor Imaging in Forensic Radiology
}

\author{
Patricia Mildred Flach, MD, *\$ Sarah Schroth, MD, $\$$ // Wolf Schweitzer, MD, * \\ Garyfalia Ampanozi, MD, * Johannes Slotboom, PhD, $\S$ Claus Kiefer, PhD, $\S$ Tanja Germerott, MD, $\$$ \\ Michael J. Thali, MD, Exec MBA, * and Marwan El-Koussy, MD
}

\begin{abstract}
Purpose: In traumatic brain injury, diffusion-weighted and diffusion tensor imaging of the brain are essential techniques for determining the pathology sustained and the outcome. Postmortem cross-sectional imaging is an established adjunct to forensic autopsy in death investigation. The purpose of this prospective study was to evaluate postmortem diffusion tensor imaging in forensics for its feasibility, influencing factors and correlation to the cause of death compared with autopsy.

Methods: Postmortem computed tomography, magnetic resonance imaging, and diffusion tensor imaging with fiber tracking were performed in 10 deceased subjects. The Likert scale grading of colored fractional anisotropy maps was correlated to the body temperature and intracranial pathology to assess the diagnostic feasibility of postmortem diffusion tensor imaging and fiber tracking.

Results: Optimal fiber tracking ( $>15,000$ fiber tracts) was achieved with a body temperature at $10^{\circ} \mathrm{C}$. Likert scale grading showed no linear correlation $(P>0.7)$ to fiber tract counts. No statistically significant correlation between total fiber count and postmortem interval could be observed $(P=0.122)$. Postmortem diffusion tensor imaging and fiber tracking allowed for radiological diagnosis in cases with shearing injuries but was impaired in cases with pneumencephalon and intracerebral mass hemorrhage.

Conclusions: Postmortem diffusion tensor imaging with fiber tracking provides an exceptional in situ insight "deep into the fibers" of the brain with diagnostic benefit in traumatic brain injury and axonal injuries in the assessment of the underlying cause of death, considering influencing factors for optimal imaging technique.
\end{abstract}

Key Words: diffusion tensor imaging, DTI, forensic radiology, magnetic resonance imaging, postmortem MR, Virtopsy

(Am J Forensic Med Pathol 2015;36: 153-161)

Traun raumatic brain injury (TBI) is a public health problem with prevalent fatal outcomes. Rates of TBI-related deaths are correlated with incidents involving firearms, motor vehicle accidents, and falls. ${ }^{1}$ Traumatic brain injury is a leading cause of death

Manuscript received January 29, 2015; accepted April 28, 2015.

From the *Institute of Forensic Medicine, University of Zurich; and $\dagger$ Department of Diagnostic and Interventional Radiology, University Hospital of Zurich, Zurich; and \$Institute of Forensic Medicine, University of Bern; and Departments of §Interventional and Diagnostic

Neuroradiology and $\|$ Neurology, University Hospital of Bern, Inselspital, Bern, Switzerland; and Institute of Legal Medicine, Medical School Hannover, Germany.

The authors report no conflict of interest.

P.M.F.: manuscript writing, study organization, image interpretation; S.S manuscript writing and editing; W.S. and G.A.: statistics, forensic integrity; J.S.: technical MR parameters, manuscript editing; C.K.: technical MR parameters, manuscript editing; T.G.: editing, study organization; M.J.T.: study design; M.E.: manuscript writing, image interpretation.

Reprints: Patricia Mildred Flach, MD, Institute of Forensic Medicine, Virtopsy, University of Zurich, Winterthurerstrasse 190/52, 8057 Zurich,

Switzerland. E-mail: patricia.flach@irm.uzh.ch.

Copyright (C) 2015 Wolters Kluwer Health, Inc. All rights reserved.

ISSN: 0195-7910/15/3603-0153

DOI: 10.1097/PAF.0000000000000177 with at least 1.7 million people sustaining TBI annually resulting in approximately 53,000 deaths in the United States. ${ }^{2}$ Traumatic brain injury is a contributing factor for almost a third of all injury-related deaths in the United States. ${ }^{2}$

In forensic pathology, death due to central regulatory failure may be the result of primary functional failure due to an intracranial process, such as intoxication, mass effect (eg, hemorrhage, edema), ischemia, injury to vasculature, and TBI, or a secondary failure, such as brainstem hemorrhage (Duret bleeding), spinal shock, or brainstem herniation. In addition, extracranial processes can lead to central regulatory failure due to exsanguination/ ensanguination, hyperthermia and hypoglycemia, and toxins. ${ }^{3}$ The majority of cases for central regulatory failure in forensics result from accidental injuries with severe head trauma or intoxication. $^{3-5}$

In clinical practice, diffusion-weighted magnetic resonance imaging has become routine for the evaluation of brain parenchyma and specifically for the assessment of white matter in living subjects. ${ }^{6,7}$ Conventional magnetic resonance imaging allows for the assessment of macrostructural morphology and pathologies. Diffusion tensor imaging (DTI) adds information about the microstructural changes within the white matter using fiber tractography. Diffusion tensor imaging coupled with tractography allows virtual dissections of white matter pathways in the living human brain and noninvasively provides maps of microscopic structural information in vivo. 8,9

Because radiological imaging became routine in forensic investigation with the application of postmortem computed tomography (PMCT), PMCT angiography, and postmortem magnetic resonance (PMMR), additional technologies such as molecular magnetic resonance and diffusion-weighted imaging are being explored for their diagnostic benefit. ${ }^{10-23}$ The purpose of this study was to evaluate the feasibility of postmortem DTI (PMDTI) in forensics, as well as its influencing factors and correlation to the cause of death compared with autopsy.

\section{MATERIALS AND METHODS}

\section{Subjects}

Both the appropriate justice department and ethics committee of the University of Bern, Switzerland approved this study. A consecutive prospective evaluation of 10 deceased cases was performed from April 2010 to January 2011. The study population consisted of 8 males and 2 females with an age range from 15 to 71 years (mean, 44.2 years; median, 46.5 years). All cases underwent whole-body PMCT, followed by PMMR of the brain and subsequent autopsy. Exclusion criteria were vast destruction of the head, face, and spine; homicide; progressed decomposition; frozen or charred bodies; cases with a history of neurological disorder; or prior PMCT angiography procedures (to avoid alterations to the imaging caused by postmortem contrast media mixture). 
Five subjects presented with TBI $(\mathrm{n}=5,4$ males and 1 female [age range, 15-65 years; mean, 37.2 years; median, 33 years]), and 5 presented without TBI $(n=5,4$ males and 1 female [range, 4271 years; mean, 51.2 years; median, 48 years]). All cases within the TBI group presented with accident as cause of death distributed within cardiac arrest $(\mathrm{n}=2)$, exsanguination $(\mathrm{n}=1)$, and central regulatory failure $(\mathrm{n}=2)$. In 1 case, cardiac arrest caused myocardial infarction and an agonal fall with extensive TBI. The other case sustained a severe traumatic fat embolism (Falzi grading II-III) with right-sided heart failure, also with extensive TBI. ${ }^{24,25}$ The group without TBI included 2 cases with intoxication in either an accidental or suicidal manner, along with consequent central regulatory failure. Myocardial infarction was detected in 2 cases and spontaneous drug-induced intracranial bleeding in 1 case.

\section{Postmortem CT Imaging}

Routine postmortem whole-body imaging was performed on a 6-slice CT scanner (Somatom Emotion 6; Siemens Medical, Erlangen, Germany) with a dedicated, on-site scanner. Raw data acquisition was performed with the following settings: collimation $6 \times 1-\mathrm{mm}$ whole body with $130 \mathrm{kV}, 160 \mathrm{~mA}$. Only images of the head were evaluated for this study; the brain tissue and bone weighted reconstruction kernel was used for image reconstruction. Slice thicknesses were 5 and $1.25 \mathrm{~mm}$ with an increment of half the slice thickness.

\section{Postmortem MR Imaging}

Imaging of the brain in situ was performed on a clinical 3-T MR scanner (Siemens Magnetom Verio; Siemens Medical) using a quadrature head coil after the clinical routine. The body was wrapped in an artifact-free body bag to avoid unwanted contamination of the clinical scanner and to ensure anonymity. The postmortem interval between death and PMMR ranged from 13 to maximal 144 hours (mean, 39.1 hours; median, 19 hours).

Standard protocol included the following sequences for macrostructural assessment: T1-weighted magnetization prepared rapid gradient echo with isotropic voxel size (sagittal, $1 \times 1 \times 1 \mathrm{~mm}$, 160 slices, field of view [FoV] $256 \mathrm{~mm}$, repetition time [TR]/time to echo [TE] 2300/2.98 milliseconds), T2 weighted (TR/TE 5000/ 95 milliseconds) and proton density (TR/TE 3000/21)-weighted turbo spin echo (axial, 24 slices, $5 \mathrm{~mm}$, FoV 512), turbo inversion recovery magnitude (coronal, 42 slices, $4 \mathrm{~mm}$, TR/TE 8500/81 milliseconds, FoV 320), constructive interference in steady state (sagittal, $0.9 \times 0.5 \times 0.6 \mathrm{~mm}, 56$ slices, FoV 240, TR/TE 1000/134 milliseconds), and high-resolution susceptibility-weighted image (axial, $1.2 \mathrm{~mm}, 120$ slices, FOV 240, TR/TE 28/20 milliseconds) with calculated maximum intensity projection images (113 slices).

Diffusion tensor imaging data in 2-dimensional single-shot EPI sequence was acquired in an axial orientation in $2.2 \times$ $2.2 \times 2.2-\mathrm{mm}$ resolution with diffusion weighting, a b1 value of $0 \mathrm{~s} / \mathrm{mm}^{2}$, and a b2 value of $1300 \mathrm{~s} / \mathrm{mm}^{2}$. Imaging parameters include TR/TE 10100/97 milliseconds, bandwidth 1346 Hz/Px, 55 slices, FoV $280 \mathrm{~mm}$, and partial Fourier factor 6/8. Images were acquired in 12 diffusion-encoding directions. The total scanning time was 60 minutes.

\section{Temperature}

Depending on the duration of the cooling time at $4^{\circ} \mathrm{C}$, body core temperature varied from 6.2 to $35.4^{\circ} \mathrm{C}$ (mean, $18.2^{\circ} \mathrm{C}$; median, $16.7^{\circ} \mathrm{C}$ ). Body core temperature was measured before and after PMMR by placing a digital thermometer deep in the rectum of the corpse. After PMMR, the body core temperature showed a range from 7.5 to $33.4^{\circ} \mathrm{C}$ (mean, $18.3^{\circ} \mathrm{C}$; median, $16.8^{\circ} \mathrm{C}$ ).
Ambient air temperature supply (range, $25.7^{\circ} \mathrm{C}-26.1^{\circ} \mathrm{C}$; mean, $25.9^{\circ} \mathrm{C}$; median, $25.9^{\circ} \mathrm{C}$ ) and rebate (range, $29.6^{\circ} \mathrm{C}-30.2^{\circ} \mathrm{C}$; mean, $29.8^{\circ} \mathrm{C}$; median, $29.7^{\circ} \mathrm{C}$ ) in the MR scanner room were measured. The mean ambient air temperature was $27.9^{\circ} \mathrm{C}$ (median, $27.8^{\circ} \mathrm{C}$ ). The correlation between ambient air temperature, body core temperature, and its effects on the cooling or warming of each body was evaluated.

\section{Evaluation of Imaging}

A picture archiving and communication system workstation (OsiriX, version 4.1.1, 64-bit; Pimeo, Geneva, Switzerland) was used for image review. Two board-certified radiologists performed image interpretation: 1 additionally board certified in neuroradiology and the other trained in postmortem imaging and forensics.

Data analysis for PMDTI was performed both on a Leonardo postprocessing workstation (Syngo software; Siemens Medical Solutions) and by an image processing and analysis software application nordicICE Diffusion/DTI Module (version 2.3.6; Nordic Neuro Lab, Bergen, Norway). In all cases, axonal tracts (fiber tracking) in the central nervous system were reconstructed for visualizing white matter connectivity. In each case, all fiber tracts were calculated and specific regions of interest such as the corpus callosum, corticospinal tracts, and bilateral superior/inferior longitudinal fasciculus. Finally, underlay volumes, such as the structural T1-weighted isotropic sequence and/or the color-coded eigenvector fractional anisotropy (FA) map, were used to superimpose the 3-dimensional visualization of white matter fiber tracts for better spatial orientation. ADC maps were not specifically assessed in the underlying study.

\section{Autopsy}

Two forensic pathologists, at least 1 of whom was board certified, performed an external inspection and conventional autopsy with standard dissection of all 3 body cavities after imaging in each case. The investigating forensic pathologist performed a neuropathological evaluation and detailed documentation of macroscopic pathologies. The forensic radiologist was present at each autopsy (after radiological report) for extended documentation (photography). Each brain was removed in toto, and in 7 cases, initial dissection was performed horizontal to the largest diameter (Flechsig cut) with coronal slices of the upper and lower part of the brain (approximate thickness of $1 \mathrm{~cm}$ ). In the remaining 3 cases, which had multiple parenchymal bleedings, coronal slices (approximate thickness of $1 \mathrm{~cm}$ ) of the whole brain were dissected to conform to findings from intracranial images. The postmortem interval between death and autopsy ranged from 26 to 158 hours (mean, 52.5 hours; median, 34.5 hours). Autopsy served as the reference standard for a direct comparison to preautopsy radiological findings on PMCT, PMMR, and PMDTI. Histology was not performed.

\section{Statistical Analysis}

The 2 radiologists assessed the image quality of the colorcoded FA map independently, based on a 5-point Likert scale. The grading of each item was scored as $1=$ unassessable, $2=$ poor, $3=$ average, $4=$ good, or $5=$ very good. In addition, a total count of tractable fiber was obtained using the nordicICE software.

The intraclass correlation coefficient based on Likert scale grading was calculated, and the results from each radiologist were pooled and correlated to the actual total of all calculated fiber tracts and the dependency of the amount of tracts and image quality to the mean body core temperature (pooled temperature before and after PMMR). In addition, the correlation of influencing factors, such as hemorrhage or pneumencephalon, the amount 
of observed tracts, and Likert scores, to the cause of death was evaluated.

White mater connectivity in the regions of interest was investigated by the correlation of fiber disruption on tractography to macrostructural images (PMCT and PMMR) and to autopsy. Finally, white matter disruption, determined by tractography, was correlated to the cause of death and the interval between death and imaging. Statistical analyses were performed using the Pearson or Spearman linear correlation test. The evaluations were performed with the statistics R-package (R Development Core Team, R: A Language and Environment for Statistical Computing, R Foundation for Statistical Computing, 2010) and JMP (SAS, JMP, version 9; SAS Institute Inc, Cary, NC).

\section{RESULTS}

\section{Temperature}

Half of the cases $(n=5)$ showed an increase in temperature after PMMR with a temperature range from $+0.8^{\circ} \mathrm{C}$ to $+2.4^{\circ} \mathrm{C}$ (mean, $+1.4^{\circ} \mathrm{C}$ ), whereas the other 5 cases showed a decrease of temperature with a range from $-0.4^{\circ} \mathrm{C}$ to $-1.5^{\circ} \mathrm{C}$ (mean, $-1.1^{\circ} \mathrm{C}$ ). The temperature cutoff for warming the body was less than a mean body temperature of $13.8^{\circ} \mathrm{C}$ (before PMMR: $12.9^{\circ} \mathrm{C}$, after PMMR: $14.7^{\circ} \mathrm{C}$ ), and for cooling, greater than $19.7^{\circ} \mathrm{C}$ (before PMMR: $20.4^{\circ} \mathrm{C}$, after PMMR: $18.9^{\circ} \mathrm{C}$ ), considering a mean temperature of $27.8^{\circ} \mathrm{C}$ in the MR suite with a scanning time of 1 hour. This results in a cutoff of $16.7^{\circ} \mathrm{C}$, leading to the cooling of corpses with a body temperature greater than $16.7^{\circ} \mathrm{C}$ or the warming of corpses with a body temperature less than $16.7^{\circ} \mathrm{C}$, assuming stable environmental conditions.

\section{Morphological Findings on Imaging Compared With Autopsy}

One case (TBI, case 5) displayed distinct pneumencephalon, acting as a major influencing factor for PMDTI and fiber tracking. Overall, autopsy missed findings such as the described pneumencephalon $(n=1)$, fractures of the viscerocranium $(n=2)$, minimal agonal intraventricular subarachnoid hemorrhage (SAH, $\mathrm{n}=1)$, shearing injuries in the cerebrum $(\mathrm{n}=1)$, and in the brainstem $(\mathrm{n}=1)$ when correlated to postmortem conventional imaging. However, autopsy detected galeal hematoma $(\mathrm{n}=1)$, slight subdural hematoma $(\mathrm{SDH}, \mathrm{n}=1)$, and cortical contusion $(\mathrm{n}=1)$ where imaging did not display such findings. Postmortem CT, when correlated to autopsy and PMMR, did not reveal cerebral contusions $(n=2)$, brainstem shearing injuries $(n=1)$, and lacunar infarction $(\mathrm{n}=1)$.

\section{Body Temperature and Fiber Tracks}

Comparing the core body temperature with the total count of tractable fibers on PMDTI showed that more favorable results for fiber tracking $(>15,000$ counted fiber tracts) were achieved when the body temperature was approximately $10^{\circ} \mathrm{C}$, than in bodies with higher or lower temperatures (Table 1). The age of the deceased correlated negatively to the fiber tracking count $(P<0.001)$.

\section{Postmortem Interval and Fiber Tracks}

There was no linear correlation between the total count of fiber tracks and the time of death to imaging interval $(P=0.122$, Spearman test of linear correlation). The mean fiber track count in all cases was 12,842.1 (range, 3097-22,929).
TABLE 1. A: Fiber track count ( $y$ axis) correlates with the mean body temperature $\left(x\right.$ axis $\left[{ }^{\circ} \mathrm{C}\right]$ ) and age of the deceased ( $z$ axis [years]). Bodies with core temperatures of approximately $10^{\circ} \mathrm{C}$ allow for a higher fiber tracking count than bodies at higher or lower temperatures. The age of the deceased correlates negatively with the fiber tracking count. The diagram shows a 3-dimensional scatterplot together with 10th (blue) and 50th (red) percentile 3-dimensional contour quantiles. B: Comparing the mean body core temperature ( $x$ axis) against the total PMDTI fiber track count ( $y$ axis), fitting a smooth spline (lambda $=10, R^{2}=0.64$ ), an optimal fiber track yield of more than 15,000 fiber tracks will be at approximately $10^{\circ} \mathrm{C}$.

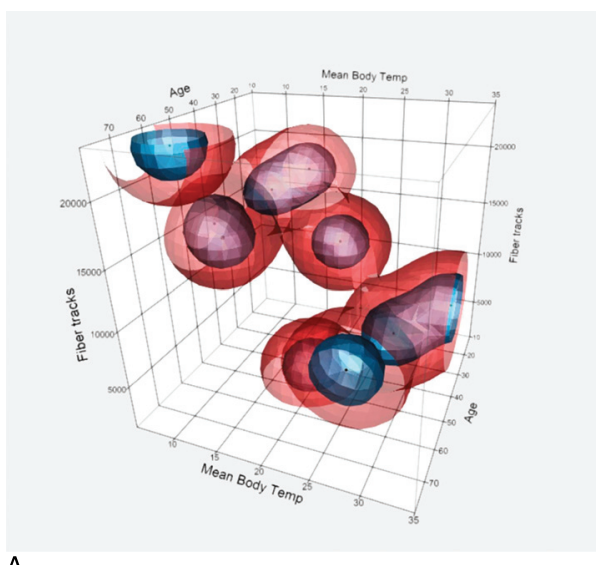

A

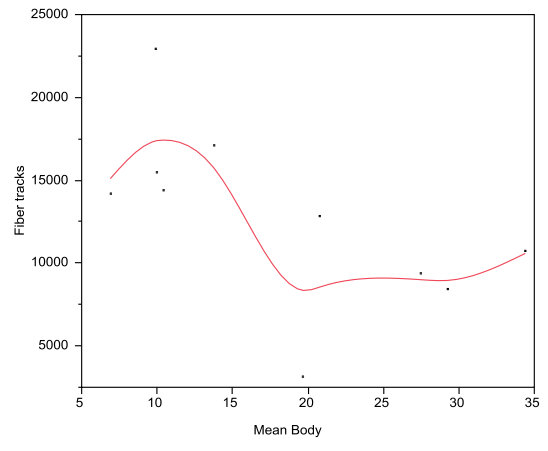

\section{Likert Scale Assessment on Colored FA Maps}

Image quality assessment of the color-coded FA map based on a 5-point Likert scale yielded only 1 case with a very good grading. The mean Likert scale grading revealed cases with ratings of good $(n=3)$, average $(n=3)$, and poor to almost unassessable $(n=3)$ (Table 2). The intraclass correlation coefficient of the Likert scores showed a good agreement $(0.855$; $95 \%$ confidence interval, $0.51-0.97){ }^{26}$ Statistical analysis revealed no apparent linear correlation between the fiber track count and Likert scale grading $(P>0.7)$ and a weak trend between the body core temperature and the Likert scale grading $(P>0.25)$. Higher Likert scale ratings correlated with a higher probability for the absence of intracranial hemorrhage in logistic regression $(P=0.08$, not statistically significant), implying a trend for better visual assessment if no hemorrhage was present. The lowest rating was found in the deceased (case 10) with pontine and massive intraparenchymal 
TABLE 2. Summary of the Morphological Findings During Autopsy, on PMCT and PMMR

\begin{tabular}{|c|c|c|c|c|c|c|c|c|c|c|c|c|}
\hline & & & Case 1 & Case 2 & Case 3 & Case 4 & Case 5 & Case 6 & Case 7 & Case 8 & Case 9 & Case 10 \\
\hline TBI & & & - & - & - & - & + & + & + & + & + & - \\
\hline \multirow{2}{*}{ Pneumencephalon } & Autopsy & & - & - & - & - & - & - & - & - & - & - \\
\hline & PMCT & & - & - & - & - & + & - & - & - & - & - \\
\hline \multirow{4}{*}{ Galeal hematoma } & \begin{tabular}{|l|} 
PMMR \\
\end{tabular} & & - & - & - & - & + & - & - & - & - & - \\
\hline & Autopsy & & - & - & + & - & + & + & + & + & + & - \\
\hline & PMCT & & - & - & + & - & + & + & + & + & - & - \\
\hline & PMMR & & - & - & + & - & + & + & + & + & - & - \\
\hline \multirow{3}{*}{ Skull fracture } & Autopsy & & - & - & - & - & + & - & - & - & - & - \\
\hline & PMCT & & - & - & - & - & + & - & - & - & - & - \\
\hline & PMMR & & - & - & - & - & + & - & - & - & - & - \\
\hline \multirow{3}{*}{ Facial fracture } & Autopsy & & - & - & - & - & - & - & - & - & - & - \\
\hline & PMCT & & - & - & - & - & - & + & + & - & - & - \\
\hline & \begin{tabular}{|l|} 
PMMR \\
\end{tabular} & & - & 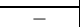 & - & - & - & + & + & - & - & - \\
\hline \multirow{3}{*}{ Brain edema } & Autopsy & & + & + & - & - & - & - & - & + & - & + \\
\hline & PMCT & & + & + & - & - & - & - & - & + & - & + \\
\hline & \begin{tabular}{|l|} 
PMMR \\
\end{tabular} & & + & + & - & - & - & - & - & + & - & + \\
\hline \multirow{3}{*}{ SAH } & Autopsy & & - & - & - & - & + & + & + & + & + & + \\
\hline & PMCT & & - & - & + & - & + & + & + & + & + & + \\
\hline & \begin{tabular}{|l|} 
PMMR \\
\end{tabular} & & - & - & + & - & + & + & + & + & + & + \\
\hline \multirow{3}{*}{$\mathrm{SDH}$} & Autopsy & & - & - & - & - & - & + & + & - & - & + \\
\hline & PMCT & & - & - & - & - & - & - & + & - & - & + \\
\hline & \begin{tabular}{|l|} 
PMMR \\
\end{tabular} & & - & - & - & - & - & - & + & - & - & + \\
\hline \multirow{3}{*}{ EDH } & Autopsy & & - & - & - & - & - & - & - & - & - & - \\
\hline & PMCT & & - & - & - & - & - & - & - & - & - & - \\
\hline & \begin{tabular}{|l|} 
PMMR \\
\end{tabular} & & - & - & - & - & - & - & - & - & - & - \\
\hline \multirow{9}{*}{ ICB cerebrum } & Autopsy & \multirow{3}{*}{ Parenchyma } & - & - & - & - & - & - & - & - & - & + \\
\hline & PMCT & & - & - & - & - & - & - & - & - & - & + \\
\hline & \begin{tabular}{|l|} 
PMMR \\
\end{tabular} & & - & - & - & - & - & - & - & - & - & + \\
\hline & Autopsy & \multirow{3}{*}{ Contusion } & - & - & - & - & + & - & + & + & - & - \\
\hline & PMCT & & - & - & - & - & - & - & - & - & - & - \\
\hline & \begin{tabular}{|l|} 
PMMR \\
\end{tabular} & & - & - & - & - & - & - & + & + & - & - \\
\hline & Autopsy & \multirow{3}{*}{ Shearing } & - & - & - & - & - & - & - & + & - & - \\
\hline & \begin{tabular}{|l|} 
PMCT \\
\end{tabular} & & - & - & - & - & - & - & - & + & - & - \\
\hline & \begin{tabular}{|l} 
PMMR \\
\end{tabular} & & - & - & - & - & - & - & + & + & - & - \\
\hline ICB cerebellum & Autopsy & Parenchyma & - & - & - & - & - & - & + & - & - & - \\
\hline & PMCT & & - & - & - & - & - & - & + & - & - & + \\
\hline & \begin{tabular}{|l|} 
PMMR \\
\end{tabular} & & - & - & - & - & - & - & + & - & - & + \\
\hline & Autopsy & Contusion & - & - & - & - & - & - & - & - & - & - \\
\hline & PMCT & & - & - & - & - & - & - & - & - & - & - \\
\hline & \begin{tabular}{|l|} 
PMMR \\
\end{tabular} & & - & - & - & - & - & - & - & - & - & - \\
\hline & Autopsy & Shearing & - & - & - & - & - & - & - & - & - & - \\
\hline & PMCT & & - & - & - & - & - & - & - & - & - & - \\
\hline & PMMR & & - & - & - & - & - & - & - & - & - & - \\
\hline ICB brainstem & Autopsy & Parenchyma & - & - & - & - & - & - & - & - & - & + \\
\hline & PMCT & & - & - & - & - & - & - & - & - & - & + \\
\hline & \begin{tabular}{|l|} 
PMMR \\
\end{tabular} & & - & - & - & - & - & - & - & - & - & + \\
\hline & Autopsy & Shearing & - & - & - & - & - & - & + & - & - & - \\
\hline & PMCT & & - & - & - & - & - & - & - & - & - & - \\
\hline & \begin{tabular}{|l|} 
PMMR \\
\end{tabular} & & - & - & - & - & - & - & + & + & - & - \\
\hline Infarction & \begin{tabular}{|l|} 
Autopsy \\
\end{tabular} & & - & - & - & - & - & - & - & - & + & - \\
\hline & PMCT & & - & - & - & - & - & - & - & - & - & - \\
\hline & PMMR & & - & - & - & - & - & - & - & - & + & - \\
\hline Vascular lesion & Autopsy & & - & - & - & - & - & - & - & - & - & - \\
\hline & PMCT & & - & - & - & - & - & - & - & - & - & - \\
\hline & PMMR & & - & - & - & - & - & - & - & - & - & - \\
\hline Fiber tracks (n) & & & 14,184 & 10,729 & 3097 & 8382 & 22,929 & 12,804 & 15,478 & 17,090 & 9348 & 14,380 \\
\hline Likert scale & Rater 1 & & 4 & 4 & 2 & 3 & 3 & 5 & 3 & 3 & 4 & 2 \\
\hline & Rater 2 & & 4 & 4 & 3 & 3 & 2 & 5 & 3 & 3 & 4 & 1 \\
\hline Likert scale (mean) & & & 4 & 4 & 2.5 & 3 & 2.5 & 5 & 3 & 3 & 4 & 1.5 \\
\hline Body temperature (mean) & & & 6.9 & 34.4 & 19.7 & 29.3 & 9.9 & 20.8 & 10 & 13.8 & 27.5 & 10.4 \\
\hline
\end{tabular}

The table shows also an overview of the temperature, fiber tracking, and Likert scale rating.

Autopsy-detected findings that were missed by imaging (red); findings missed during autopsy (blue); findings missed by PMCT, but observed by PMMR and during autopsy (green).

+ Indicates positive finding; -, negative finding; ICB, intracranial bleeding; EDH, epidural hemorrhage. 
hemorrhage due to extensive susceptibility artifacts. The subject (case 5) with extensive pneumencephalon yielded a poor to average Likert scale rating on the FA map.

There was no statistically significant difference on PMDTI derived from the fiber track count and averaged Likert scores when comparing cases with and without subdural hemorrhage, intracerebral or cerebellar hemorrhage, cerebral shearing injuries, or brainstem lesions.

\section{Morphological Findings on Imaging and Autopsy Compared With Fiber Tracking}

\section{TBI Group}

The TBI group included cases 5 to 9 (Table 2). Case 5 presented with shearing injuries, skull fractures, SAH on imaging and gross pathology, and small cortical contusions during autopsy. Imaging showed additional pneumencephalon. The total count of fiber tracks was highest in this case $(n=22929)$ at a mean body temperature of $9.9^{\circ} \mathrm{C}$. Fiber tracking was compromised by the pneumencephalon, $\mathrm{SAH}$, galeal hematoma, and blood around the head in the body bag, which resulted in a mean Likert scale grading (2.5) and distortion of the corpus callosum fiber tracks predominantly on the left side (Fig. 1).

Case 6 was rated highest on the Likert scale ${ }^{5}$ and had a total fiber count of 12,804 at a body temperature of $20.8^{\circ} \mathrm{C}$. Images of the deceased revealed fractures of the viscerocranium, galeal hematoma, and slight intraventricular SAH. Despite negative findings regarding SDH by the imaging protocol, autopsy stated a fine SDH. For fiber tracking, the minimal SAH did not decrease PMDTI image quality, and only small gaps in the corticospinal tract could be detected. There were no other defects on fiber tracking noted (Fig. 2).

Case 7 was involved in a traffic accident and suffered from severe TBI with shearing injuries, SAH, SDH, cerebral contusions, cerebellar parenchymal bleeding, and galeal hematoma. The cerebral shearing injuries were initially not observed during autopsy and PMCT and were detectable only on PMMR. Autopsy and PMMR showed concordance in detection of cerebral contusions and shearing injuries in the brainstem. Fractures of the viscerocranium were detected only by imaging. The total fiber count was relatively high $(15,478)$ at a body temperature of $10^{\circ} \mathrm{C}$. The average Likert scale rating was 3 . The fiber tracking displayed concordant disruption of the fiber in the corticospinal tract (decussation of pyramids, crossing fibers) and a gap in the genu and body of the corpus callosum, according to the localization of shearing injuries (Fig. 3).

Case 8 died of TBI with exsanguination due to splenic laceration and fat embolism. Head trauma presented with galeal hematoma, brain edema, SAH, cerebral shearing injuries, and cerebral contusions. The cerebral contusions were not detected by PMCT, and the midbrain (cerebral peduncle) shearings were observed only on PMMR. The hemorrhage into the right midbrain was technically not dissected during autopsy because of a caudal transection of the brainstem at the level above the pons with transverse slices and coronal slices of the cerebrum including the undissected midbrain. The total fiber count was 17,090 at a body temperature

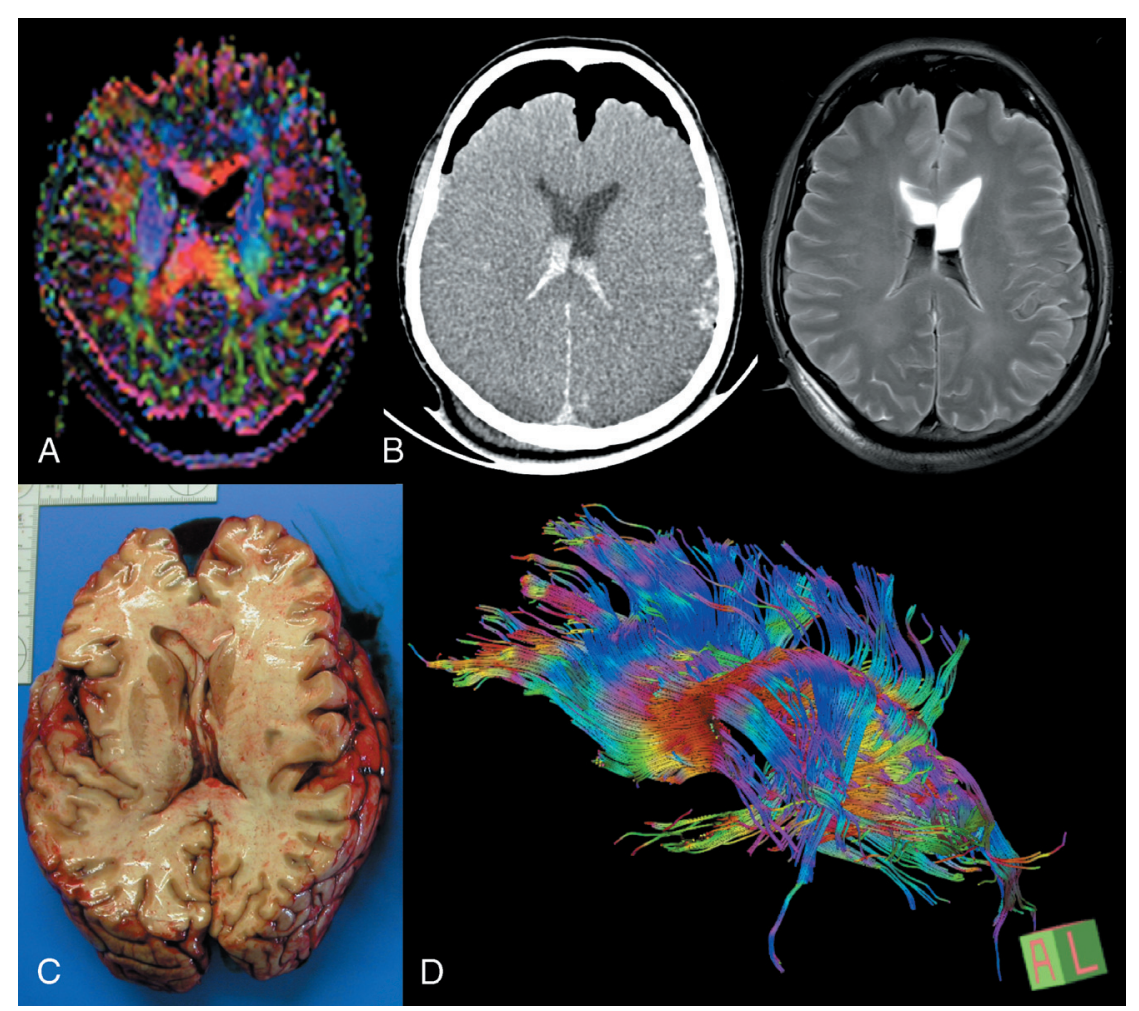

FIGURE 1. Case 5, TBI. A, Colored FA map with distortion due to compromising factors such as pneumencephalon, SAH, galeal hematoma, and blood around the head in the body bag. B, Postmortem CT displaying the extent of pneumencephalon that was not described during autopsy; note the SAH with intraventricular blood and the right occipital galeal hematoma. On the right PMMR, T2-weighted sequence, corresponding to the cross section of PMCT. C, Typical Flechsig cut of the brain in forensic autopsy at the level of the side ventricles. Detection of intraventricular blood is hardly feasible, as the fluid will flow out during removal of the brain. Note the SAH. D, This case showed the highest fiber track count at a mean body temperature of $9.9^{\circ} \mathrm{C}$. Fiber tracking was compromised by influencing factors $(A)$, resulting in a low Likert scale grading (2.5), and distortion of the fibers of the corpus callosum fiber tracks, predominantly on the left side. 
of $13.8^{\circ} \mathrm{C}$ with an average Likert grading of 3 . Fiber tracking revealed incomplete tracts of the right corticospinal tract and of the body of the corpus callosum. These findings were concordant between imaging and autopsy (except for the midbrain shearing injuries that were not validated by autopsy).

\section{Non-TBI Group}

The non-TBI group included cases 1 to 4 and 10 (Table 2). Cases 1 and 2 both died of intoxication and exhibited distinct brain edema. The total fiber counts were 14,184 and 10,729 with a Likert scale rating of 4 and body temperatures of $6.9^{\circ} \mathrm{C}$ and $34.4^{\circ} \mathrm{C}$, respectively. Postmortem magnetic resonance was impaired by the cuff of an endotracheal tube at the level of the mandible in case 1. Fiber tracking was not impaired.

Case 3 died of cardiac arrest and sustained a galeal hematoma during agonal collapse with minimal intraventricular SAH. The total fiber count was 3097 with a poor to average Likert scale grading (2.5) at a body temperature of $19.7^{\circ} \mathrm{C}$. Fiber tracking by the nordicICE software was not diagnostic; however, the Siemens software allowed for diagnostic interpretation and revealed no fiber disruption.

Case 4 revealed a natural manner of death caused by cardiac arrest without intracranial pathology. This case showed a relatively high body temperature of $29.3^{\circ} \mathrm{C}$ and a total of 8382 fiber tracks at an average Likert scale grading of 3 . Fiber tracking was feasible without gaps, disruption, or distortion. The foreign body (infusion bag) did not interfere with PMDTI.

Case 9 presented with slight SAH, chronic lacunar cerebellar infarction, and fat embolism leading to cardiac arrest. The small galeal hematoma was observed only during autopsy. The total fiber count was 9348 with a Likert grading of 4 at $27.5^{\circ} \mathrm{C}$. Fiber tracking showed no damage.
Case 10 displayed the lowest Likert scale grading (1.5) and sustained spontaneous drug-induced pontine and intracranial mass hemorrhage with brain edema, SAH, and SDH. The total count of fiber tracks was still satisfactory with $n=14380$ at a body temperature of $10.4^{\circ} \mathrm{C}$. However, PMDTI and fiber tracking were severely impaired by the amount of intracranial blood, and major distortion of the fiber tracks was present, interfering with diagnosis by DTI (Fig. 4).

\section{DISCUSSION}

In living subjects, white matter regions with reduced anisotropy are detected in the first 24 hours after TBI, and thus, DTI may aid in the detection of diffuse axonal injury (DAI) ${ }^{27}$ Cases with mild TBI white matter changes, which may not be readily apparent on conventional neuroimaging, are primarily characterized by diffuse axonal damage and not myelin damage. However, more severe injuries usually impact both the axons and the myelin. Diffusion tensor imaging is assumed to provide an objective examination for determining pathologies related to $\mathrm{TBI}^{28}$ Diffusion tensor imaging measures may thus contribute additional diagnostic information to conventional neuroimaging related to DAI and TBI- even in blast-related TBI. ${ }^{29,30}$

This study showed that PMDTI may serve as a valuable diagnostic add-on in postmortem forensic investigation in cases with TBI, exhibiting shearing injuries with DAI, and also allow for practical fiber tracking of the brain in cases without head trauma. Major limitations for the application of PMDTI are in cases with parenchymal mass hemorrhage and distinct pneumencephalon. Postmortem fiber tractography is easily visualized in a 3-dimensional fashion and helps to give context to the legal layperson.

There are numerous publications regarding DTI in clinical patients and reports on the correlation of DTI to animal tissue or

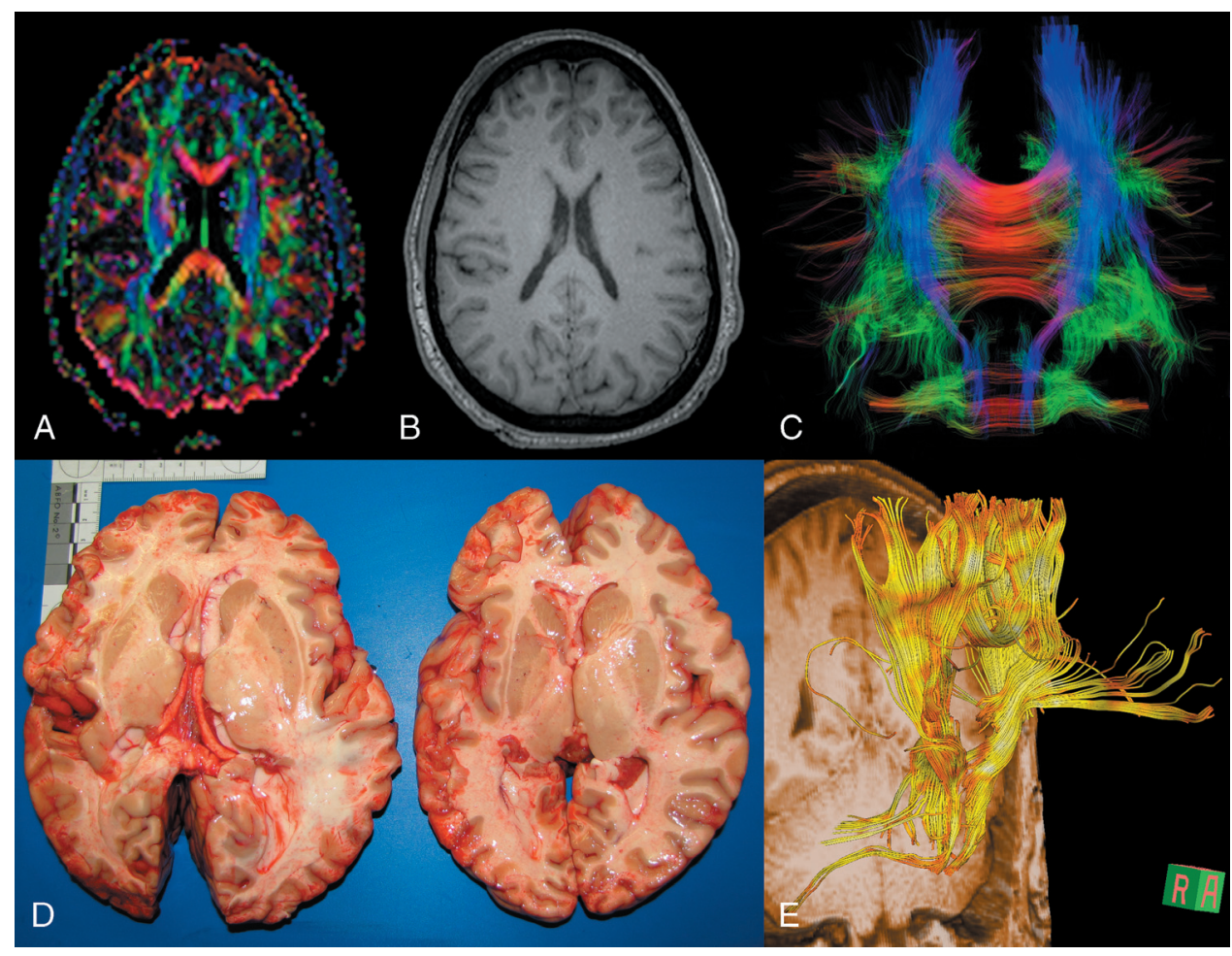

FIGURE 2. Case 6, TBI. A, The colored FA map was rated highest on the Likert scale ${ }^{5}$ with a total fiber count of 12,804 and a body temperature of $20.8^{\circ} \mathrm{C}$. Note the impeccable display of the directional colored FA. B, Isotropic T1-weighted sequence for anatomical orientation.

C, Postmortem DTI allowed for excellent depiction of the fiber tracks. D, Autopsy specimen. E, Fusion of the isotropic T1-weighted sequence and tracking of the corticospinal fibers. 


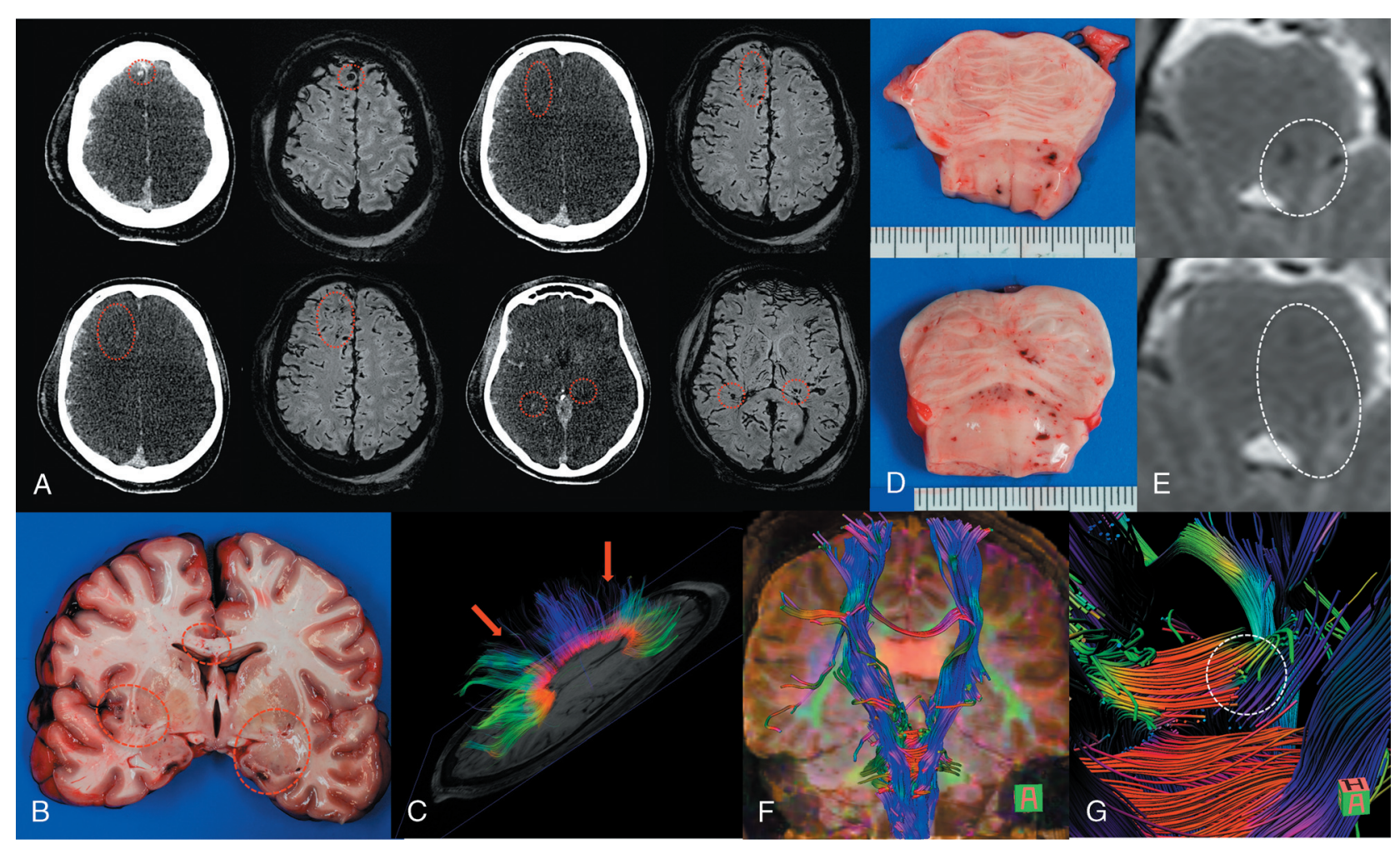

FIGURE 3. Case 7, TBI. A, Comparison of PMCT and PMMR, and SWI (susceptibility-weighted image), depicting cerebral shearing injuries (red circles). Subarachnoid hemorrhage and right-sided temporoparietal and left occipital galeal hematoma. B, Autopsy specimen of a coronal slice. The slice thickness is approximately $1 \mathrm{~cm}$, clearly not revealing all the intracranial findings because of the technique. This dissection through the brain reveals shearing injuries (red circles) in the corpus callosum and at both sides in the basal ganglia-corresponding to imaging. C, Fusion of an axial T1-weighted sequence and fiber tractography of the corpus callosum. Note the lack of fiber tracks (red arrows) at the genu and body of the corpus callosum corresponding to the location of shearing injuries on imaging. D, Autopsy specimen of the brainstem. Both images are axial cut slices of the pons. There are multiple, small dotlike hemorrhages observed, especially on the left side in the pons and extending into the cerebellar peduncle, indicating pontine shearing injuries. E, High-resolution CISS (constructive interference in steady state) sequence of the brainstem with corresponding levels to image D. According to the autopsy specimen, there is signal loss within the pons and peduncle indicating hemorrhage (white circle). F, Fusion of the anatomical T1-weighted, isotropic sequence, the colored FA map, and the fiber tractography of the pyramid with anterior view. Note the disruption of fibers at the pons. G, Magnified view from anterior to posterior of the corticospinal fibers. This image shows the disruption at the level of crossing fibers in the posterior part of the pons corresponding to the hemorrhage observed on PMMR (E) and during autopsy (D).

to ex vivo formalin-fixed human specimens that suffer considerably from changes in tissue properties. ${ }^{31-36}$ Imaging of a formalinfixed specimen results in signal alteration of the ex situ brain tissue and therefore may also alter PMDTI. If the brain is not fixed with formalin, continuing decomposition potentially impairs PMDTI, and frozen tissue as an alternative to fixation may interfere with anisotropy. Substantial literature on DTI with imaging in situ on unaltered (eg, by fixation and potential intercurrent brain damage) human postmortem brain tissue is sparse..$^{21,22}$

Postmortem DTI in situ, however, poses different problems compared with imaging the living. Temperature and decay (postmortem interval and ambient circumstances) in addition to intracranial pathologies significantly degrade the DTI quality and have a major effect on fiber tracking results and diffusionweighted imaging. According to Scheurer et al, ${ }^{22}$ in 2011 , postmortem ADC values of the brain were decreased by $49 \%$ to $72 \%$ compared with living subjects. In longer postmortem intervals, the ADC value was significantly reduced in the gray matter but not in the white matter. This led to the conclusion that postmortem ADC values may be used for the estimation of the postmortem interval, serving as crucial information in forensic examinations. ${ }^{22}$ Yen et $\mathrm{al}^{21}$ published the first study on line-scan DTI of the brainstem, investigating the brainstem in situ in 1 deceased subject and in 2 living controls. This publication proposed the potential of PMDTI and fiber tracking to provide detailed information concerning white matter tract integrity and/or disruption in cases of cervical trauma, allowing for the diagnosis of mechanical fiber tract ruptures in the postmortem subject in situ, which cannot be obtained by other imaging modalities at this point in time. ${ }^{21}$

The present study established the optimal body temperature of the corpse at approximately $10^{\circ} \mathrm{C}$ to achieve the best results for the fiber tractography on PMDTI. This result corresponds to a previous study on image contrast dependency on the temperature of a corpse on PMMR by Ruder et $\mathrm{al}^{37}$ (2012), stating that scanning of corpses less than $10^{\circ} \mathrm{C}$ should be avoided. A 60 minute-long PMMR scan affects the body temperature, leading to cooling of the corpse with a body temperature greater than $16.7^{\circ} \mathrm{C}$ or warming of the corpse at a body temperature less than $16.7^{\circ} \mathrm{C}$, assuming stable environmental conditions in the MR suite at $27.8^{\circ} \mathrm{C}$. In the living, the diffusion is directly connected to the temperature, and the colder the subject, the less water molecules move around. This may be altered in postmortem examinations because of ongoing autolysis and decay, overcompensating the temperature-related positive effects of diffusion. ${ }^{38}$ In addition, a higher signal-to-noise ratio of the images may result in more DTI fibers being tracked. A specific analysis of the signal-to-noise 


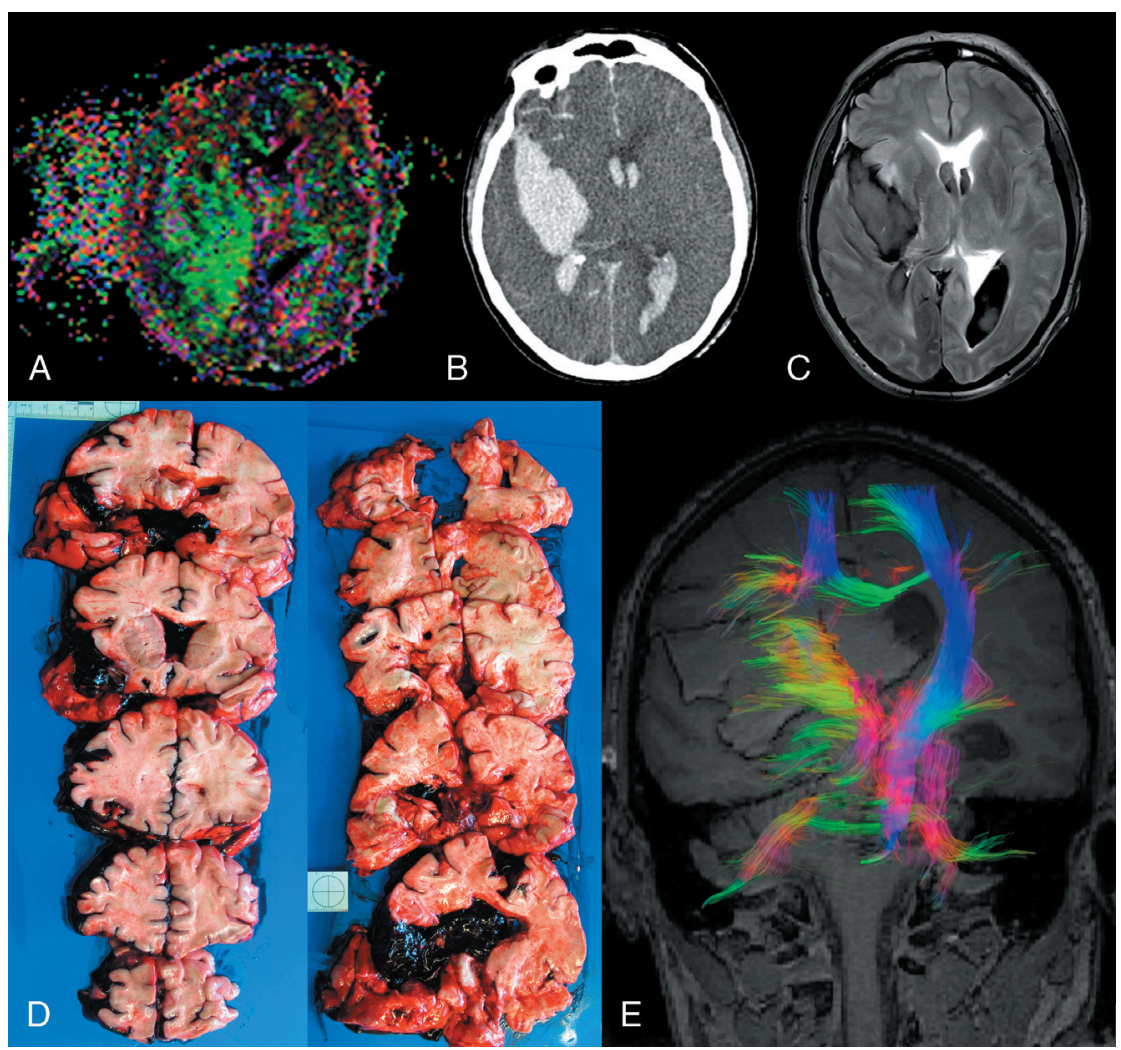

FIGURE 4. Case 10, no TBI. A, The colored FA map in this case yielded the lowest Likert scale grading (1.5). Note the vast blurring and distortion of the PMDTI. B, Postmortem CT depicting the spontaneous drug-induced pontine and intracranial mass hemorrhage extending in the ventricular system with concomitant brain edema, SAH, and slight SDH. C, T2-weighted sequence at the same level as image B. D, Autopsy specimen. The left row starts at the bottom with the frontal brain and continues in the right row at the bottom and ends at the top with coronal cuts of the occipital pole. There is a massive parenchymal hemorrhage on the right extending with predominantly coagulated blood into the ventricles. The consistency of the brain is liquefied, and the gyri and sulci are effaced according to brain edema. E, Fusion of an anatomical T1-weighted image displaying the mass hemorrhage on the right extending in the ventricles. Note the extended temporal horn on the left indicating impaired cerebrospinal fluid circulation due to the hemorrhage. The fiber tracking of the left side is still somehow feasible; however, the left side is completely distorted because of the major susceptibility of the hemorrhage.

ratio despite the Likert scale rating was not assessed. However, movement or pulsation artifacts were clearly no issue. This study showed that there was no statistically significant correlation between the fiber track count and the postmortem interval.

Knowledge of how these influencing factors alter postmortem image quality and how to optimize the PMMR and PMDTI examination may help to overcome unsatisfactory PMMR imaging results in the future. ${ }^{39-41}$

The application of PMDTI may be diagnostic not only in TBI but also in nonaccidental injuries in cases of child abuse-clearly in the living but also in the deceased. According to Oehmichen et $\mathrm{al}^{42}$ localized axonal injury was regularly present in the brains of shaken baby syndrome infants surviving longer than 1.5 to 3.0 hours, but only occasionally in the craniocervical junction. Ezaki et $\mathrm{al}^{43}$ stated that diffusion-weighted imaging cannot detect all DAI-related lesions but is a potentially useful imaging modality for both diagnosing and assessing patients with DAI.

Clearly, this study was limited because of the moderate study size and the wide range of death-to-scan time. The latter can be explained by practical logistic limitations. The study approach included cases with pneumencephalon, intracranial mass hemorrhage, and various body temperatures - all being potential biases for radiological interpretation of PMDTI. However, these factors will regularly occur if postmortem imaging is performed and were purposefully not excluded from this study. Contrarily, cases with advanced decomposition were not included and should be investigated in depth in the future-not only for the estimation of the postmortem interval but also to determine anisotropy in the decaying brain with potential diagnostic benefits in the future.

\section{CONCLUSIONS}

Postmortem cross-sectional imaging is a fairly young field and will eventually replace or at least supplement forensic autopsy. Therefore, exploring advanced techniques such as DTI is essential to achieve advancement and quality improvement in forensics. Postmortem fiber tracking with DTI is feasible, providing an exceptional insight "deep into the fibers" of the brain in situ, with potential diagnostic benefit for TBI and axonal injuries in the assessment of the underlying cause of death. Several factors, for example, body temperature of the corpse, have to be considered for optimization of the PMDTI technique.

\section{ACKNOWLEDGMENTS}

The authors thank Michela Mordasini, Carole Stuker, Timo Seip and the whole team of radiographers at the Department of Interventional and Diagnostic Neuroradiology, Inselspital, University of Bern, Switzerland for their motivated and competent assistance in imaging. Grateful thanks to Sandra Somaini for her motivated, excellent and competent assistance in imaging at the Forensic Institute, University of Bern, Switzerland. 


\section{REFERENCES}

1. Coronado VG, Xu L, Basavaraju SV, et al. Surveillance for traumatic brain injury-related deaths-United States, 1997-2007. MMWR Surveill Summ. 2011;60:1-32.

2. Get the Stats on Traumatic Brain Injury in the United State. Available at: http://www.cdc.gov/traumaticbraininjury/pdf/Bluebook_factsheet-a.pdf. Accessed December 29, 2014.

3. Saukko P, Knight B. Chapter 5: head and spinal injuries. In: Knight's Forensic Pathology. 3rd ed. London: Edward Arnold; 2004:174-221.

4. Chapter 9: closed brain injuries. In: Auer RN, König HG, Oehmichen M, eds. Forensic Neuropathology and Associated Neurology. Berlin, Germany: Springer-Verlag; 2006:177-210.

5. Chapter 6: trauma to the skull and brain: craniocerebral injuries. In:DiMaio V, DiMaio D (Eds). Forensic Pathology. 2nd ed. Boca Raton, FL: CRC Press; 2001:147-185.

6. Muir KW, Buchan A, von Kummer R, et al. Imaging of acute stroke. Lancet Neurol. 2006;5:755-768.

7. Helenius J, Soinne L, Perkiö J, et al. Diffusion-Weighted MR imaging in normal human brains in various age groups. Am J Neuroradiol. 2002;23:194-199.

8. Catani M. Thiebaut de Schotten M. A diffusion tensor imaging tractography atlas for virtual in vivo dissections. Cortex. 2008;44:1105-1132.

9. Moseley M. Diffusion tensor imaging and aging — a review. NMR Biomed. 2002;15:553-560.

10. Thali MJ, Jackowski C, Oesterhelweg L, et al. Virtopsy-the Swiss virtual autopsy approach. Leg Med (Tokyo). 2007;9:100-104.

11. Thali MJ, Yen K, Schweitzer W, et al. Virtopsy a new imaging horizon in forensic pathology: virtual autopsy by postmortem multislice computed tomography (MSCT) and magnetic resonance imaging (MRI) - a feasibility study. J Forensic Sci. 2003;48:386-403.

12. The Virtopsy Project. Available at: www.virtopsy.com. Accessed December 29, 2014.

13. Roberts IS, Benamore RE, Benbow EW, et al. Post-mortem imaging as an alternative to autopsy in the diagnosis of adult deaths: a validation study. Lancet. 2012;379:136-142.

14. Christe A, Flach P, Ross S, et al. Clinical radiology and postmortem imaging (Virtopsy) are not the same: specific and unspecific postmortem signs. Leg Med (Tokyo). 2010;12:215-222.

15. Flach PM, Ross SG, Thali MJ. Clinical and forensic radiology are not the same. In: Thali MJ, Viner MD, Brogdon BG, eds. Brogdon's Forensic Radiology. 2nd ed. Boca Raton, FL: CRC Press, Taylor \& Francis Group; 2010:409-440

16. Ross SG, Thali MJ, Bolliger S, et al. Sudden death after chest pain: feasibility of virtual autopsy with postmortem CT angiography and biopsy. Radiology. 2012;264:250-259.

17. Grabherr S, Djonov V, Yen K, et al. Postmortem angiography: review of former and current methods. AJR Am J Roentgenol. 2007;188:832-838.

18. Jackowski C, Persson A, Thali MJ. Whole body postmortem angiography with a high viscosity contrast agent solution using poly ethylene glycol as contrast agent dissolver. J Forensic Sci. 2008;53:465-468.

19. Ross S, Ebner L, Flach P, et al. Postmortem whole-body MRI in traumatic causes of death. AJR Am J Roentgenol. 2012;199:1186-1192.

20. Yen K, Lövblad KO, Scheurer E, et al. Post-mortem forensic neuroimaging: correlation of MSCT and MRI findings with autopsy results. Forensic Sci Int. 2007;15;173:21-35.

21. Yen K, Weis J, Kreis R, et al. Line-scan diffusion tensor imaging of the posttraumatic brain stem: changes with neuropathologic correlation. Am J Neuroradiol. 2006;27:70-73.

22. Scheurer E, Lovblad KO, Kreis R, et al. Forensic application of postmortem diffusion-weighted and diffusion tensor MR imaging of the human brain in situ. Am J Neuroradiol. 2011;32:1518-1524.
23. Ith M, Scheurer E, Kreis R, et al. Estimation of the postmortem interval by means of ${ }^{1} \mathrm{H}$ MRS of decomposing brain tissue: influence of ambient temperature. NMR Biomed. 2011;24:791-798.

24. Flach PM, Ross SG, Bolliger SA, et al. Massive systemic fat embolism detected by postmortem imaging and biopsy. J Forensic Sci. 2012;57: 1376-1380.

25. Filograna L, Bolliger SA, Spendlove D, et al. Diagnosis of fatal pulmonary fat embolism with minimally invasive virtual autopsy and post-mortem biopsy. Leg Med (Tokyo). 2010;12:233-237.

26. Shrout PE, Fleiss JL. Intraclass correlation: uses in assessing rater reliability. Psychol Bull. 1979;86:420-428.

27. Arfanakis K, Haughton VM, Carew JD, et al. Diffusion tensor MR imaging in diffuse axonal injury. Am J Neuroradiol. 2002;23:794-802.

28. Kraus MF, Susmaras T, Caughlin BP, et al. White matter integrity and cognition in chronic traumatic brain injury: a diffusion tensor imaging study. Brain. 2007;130(pt 10):2508-2519.

29. Niogi SN, Mukherjee P, Ghajar J, et al. Extent of microstructural white matter injury in postconcussive syndrome correlates with impaired cognitive reaction time: a $3 \mathrm{~T}$ diffusion tensor imaging study of mild traumatic brain injury. Am J Neuroradiol. 2008;29:967-973.

30. Mac Donald CL, Johnson AM, Cooper D, et al. Detection of blast-related traumatic brain injury in U.S. military personnel. $N$ Engl J Med. 2011;364:2091-2100

31. Pfefferbaum A, Sullivan EV, Adalsteinsson E, et al. Postmortem MR imaging of formalin-fixed human brain. Neuroimage. 2004;21:1585-1595.

32. Englund E, Sjöbeck M, Brockstedt S, et al. Diffusion tensor MRI post mortem demonstrated cerebral white matter pathology. J Neurol. 2004;251: $350-352$.

33. Hansen B, Flint JJ, Heon-Lee C, et al. Diffusion tensor microscopy in human nervous tissue with quantitative correlation based on direct histological comparison. Neuroimage. 2011;57:1458-1465.

34. Miller KL, Stagg CJ, Douaud G, et al. Diffusion imaging of whole, post-mortem human brains on a clinical MRI scanner. Neuroimage. 2011;57:167-181

35. D'Arceuil H, de Crespigny A. The effects of brain tissue decomposition on diffusion tensor imaging and tractography. Neuroimage. 2007;36:64-68.

36. Kim JH, Trinkaus K, Ozcan A, et al. Postmortem delay does not change regional diffusion anisotropy characteristics in mouse spinal cord white matter. NMR Biomed. 2007;20:352-359.

37. Ruder TD, Hatch GM, Siegenthaler L, et al. The influence of body temperature on image contrast in post mortem MRI. Eur J Radiol. 2012;81:1366-1370.

38. Dellanoy J, Ching-Nien C, Turner R, et al. Noninvasive temperature imaging using diffusion MRI. Magn Reson Med. 1991;19:333-339.

39. Ruder TD, Thali MJ, Hatch GM. Essentials of forensic post-mortem MR imaging in adults. Br J Radiol. 2014;87:20130567.

40. Kobayashi T, Isobe T, Shiotani S, et al. Postmortem magnetic resonance imaging dealing with low temperature objects. Magn Reson Med Sci. 2010;9:101-108.

41. Kobayashi T, Shiotani S, Kaga K, et al. Characteristic signal intensity changes on postmortem magnetic resonance imaging of the brain. Jpn J Radiol. 2010;28:8-14.

42. Oehmichen M, Schleiss D, Pedal I, et al. Shaken baby syndrome: re-examination of diffuse axonal injury as cause of death. Acta Neuropathol. 2008;116:317-329.

43. Ezaki Y, Tsutsumi K, Morikawa M, et al. Role of diffusion-weighted magnetic resonance imaging in diffuse axonal injury. Acta Radiol. 2006;47:733-740. 